\title{
The Effect of High Intensity Interval Training Versus Steady State Aerobic Training on Skin Microvascular Reactivity in Moderately Active Young Adults: A Pilot Study
}

\author{
Yoshlin Naicker ${ }^{1} \&$ Takshita Sookan ${ }^{1}$ \\ 1 Discipline of Biokinetics, Exercise and Leisure Sciences, School of Health Sciences, University of \\ KwaZulu-Natal, South Africa \\ Correspondence: Takshita Sookan, Discipline of Biokinetics, Exercise and Leisure Sciences, University of \\ KwaZulu-Natal, Private Bag X54001, Durban, 4000, South Africa. Tel: 27-31-260-7987; Fax: 27-31-260-7903. \\ E-mail: sookan@ukzn.ac.za
}

Received: June 20, 2019 Accepted: July 13, 2019 Online Published: September 2, 2019

doi:10.5539/gjhs.v11n11p11 URL: https://doi.org/10.5539/gjhs.v11n11p11

\begin{abstract}
A sedentary lifestyle is associated with endothelial dysfunction, leading to vascular pathology and impaired microvascular reactivity (MVR). Enhanced endothelial functioning has been seen in aerobically trained individuals. The purpose of this study was to determine if there was a differential training response of skin MVR in response to high intensity aerobic interval training (HIAIT) as compared to steady state aerobic training (SSAT). The study involved 16 moderately active male students (age: $20.93 \pm 5.05 \mathrm{yrs}$ ). They were randomly assigned to one of three groups; HIAIT, SSAT, and control group (CG). Baseline testing was performed to determine the $\mathrm{VO}_{2 \max }$, lactate threshold, blood pressure, body composition and a post-occlusive reactive hyperemia response (PORH) test was used to assess microvascular reactivity on the right palmer forearm using a moorVMS-Laser Doppler Flowmetry (LDF) These parameters were reassessed after three weeks (mid-point) and six-weeks (post intervention) weeks. Statistical significance was set at $p \leq 0.05$. There were no significant interactions between the variables measured in the three groups over time. There was a positive linear relationship in the SSAT group for PORHmax/time to peak $\left(\mathrm{T}_{\mathrm{p}}\right)$ at baseline $(\mathrm{r}=0.998, \mathrm{p}=0.039)$, midpoint $(\mathrm{r}=0.992, \mathrm{p}=0.083)$, and the post intervention $(\mathrm{r}=0.987, \mathrm{p}=0.103)$. Training in the SSAT group had improved $\mathrm{PORH}_{\text {peak }}$ at midpoint and post intervention time points $(\mathrm{r}=0.999, \mathrm{p}=0.22 ; \mathrm{r}=1, \mathrm{p}=0.006 ; \mathrm{r}=1, \mathrm{p}=0.011)$. Training in either the HIAIT or the SSAT group had no significant effect on skin MVR in moderately active young adults. SSAT did display a positive linear relationship with $\mathrm{PORH}_{\max } \mathrm{T}_{\mathrm{p}}$, and $\mathrm{PORH}_{\text {peak, }}$, which are variables influencing skin MVR.
\end{abstract}

Keywords: sedentary lifestyle, endothelial function, laser Doppler, aerobic training

\section{Introduction}

A low cardio-respiratory fitness level has been associated with endothelial dysfunction and decreased microvascular reactivity (MVR). As a result, individuals displaying low cardio-respiratory fitness have been shown to have an increased risk of cardiovascular disease (CVD) (Roche, Edmunds, Cable, Didi, Stratton, 2008). Some of these risk factors are modifiable. These include hypercholesterolemia, cigarette smoking, hypertension and physical inactivity (Celermajer, Sorensen, Bull, Robinson, \& Deanfield, 1994). Enhanced endothelial function and enhanced microvascular reactivity is associated with training and high cardio-respiratory fitness, especially when maximal aerobic capacity is the marker of cardio-respiratory fitness (De Souza et al., 2000; Larsen \& Matchkov, 2016; Montero, 2015; Roche et al., 2008).

Laser Doppler Flowmetry (LDF), is a non-invasive method used to determine skin microvascular reactivity (MVR). Skin MVR refers to the microcirculatory response of blood flow in tissue and a decrease may lead to an increased risk of CVD (Morales et al., 2005; Rajan, Varghese, van Leeuwen, \& Steenbergen, 2008). Optical fibres are utilised to project laser light onto the tissue, and retrieve scattered light from the tissue (Morales et al., 2005). Laser Doppler flowmetry therefore measures the Doppler shift occurring in red blood cells (Rajan et al., 2008). Laser Doppler flowmetry can provide information regarding the microvascular haemodynamics in the limbs, and has been used to measure this in response to ischaemia (Tur, Yosipovitch, \& Bar-on, 1991). 
Both resistance and aerobic training have been used to improve overall health and fitness in the general and diseased populations (Horuichi \& Okita, 2012). Steady state aerobic training (SSAT) has been proven to enhance both cardiovascular health and the functional responsiveness of the vasculature (Hwang \& Lee, 2016). Steady state aerobic training has been well-documented in terms of the positive effects on endothelial function in those with coronary artery disease (Abbott et al., 2002; Clarkson et al., 1999; Hambrecht et al., 2003). Regular SSAT has also been shown to improve both resting blood pressure and endothelium-dependent vasodilatation in hypertensive individuals (Horiuchi et al., 2012; La Favor et al., 2016). Despite multiple studies proving the benefits of cardio-respiratory training, little is known regarding the effect of exercise intensity on microcirculation (Goto et al., 2003). Therefore one cannot recommend either SSAT or HIAIT without further investigation.

In terms of cardio-respiratory training, SSAT, involves continuous aerobic exercise at a fixed pace or percentage of $\mathrm{VO}_{2 \max }$ for a period of time (Gibala, Gillen, Percival, 2014). High intensity aerobic interval training (HIAIT) has been described as a training stimulus 'near maximal' oxygen uptake, or targeting $80-100 \%$ of heart rate max (Gibala et al., 2014). $\mathrm{VO}_{2 \max }$ has been shown to improve after two weeks of HIAIT training, with vascular health shown to improve after four weeks (Gibala et al., 2014; Rakobowchuk et al., 2008).

Highly endurance-trained adolescents have better endothelium-dependent vasodilatation in the forearm skin microcirculation, compared to their untrained peers (Roche et al., 2008). This study demonstrated that long-term aerobic exercise training in adolescents is associated with enhanced skin MVR (Roche et al., 2008). Vinet et al. (2018) found that any exercise modality improved flow mediated dilation in those with metabolic syndrome. Exercise modalities included high intensity resistance training, aerobic training, or a combination of the both (Vinet et al., 2018).

Goto et al. (2003) investigated the effect of intensity of exercise on endothelium dependent vasodilatation. Three groups of men from a healthy male population were made to exercise at either $25 \%$ of their $\mathrm{VO}_{2 \max }, 50 \%$ of $\mathrm{VO}_{2 \max }$, or $75 \%$ of $\mathrm{VO}_{2 \max }$. Results showed that the group who trained at $50 \%$ of their $\mathrm{VO}_{2 \max }$ improved acetylcholine-induced vasodilatation the most, compared to the groups who trained at $25 \%$ and $75 \%$ of their $\mathrm{VO}_{2 \max }$; which had little to no further improvement in their vascular health (Goto et al., 2003). These intensities, however, were applied to SSAT. A study, focusing on four weeks of cycling in healthy males, showed improved vascular function (Tinken, Thijssen, Black, Cable, Green, 2008). Improved vascular function was also proven in another study which looked at ten weeks of daily aerobic and anaerobic training in military recruits (Tinken et al., 2008).

The effect of training modalities, such as HIAIT and SSAT, on skin MVR is unclear, as is the relationship between skin MVR and submaximal measures of cardio-respiratory fitness. As a result, more studies are needed to clarify the effects of different intensities, methods and duration of exercise on skin MVR health. Therefore, the aim of this study was to identify the effect of training intensity on skin MVR over a six-week training period.

\section{Methods}

\subsection{Study Design and Participant Recruitment}

The study design was an epidemiological and randomized experimental study. The study consisted of 25 male participants between the ages of 18 and 27. All the participants were students recruited from the University of KwaZulu-Natal, Westville campus. Participation in the study was voluntary. The study obtained ethical clearance from the University of KwaZulu Natal's Biomedical Research Ethics Committee (BE506/14). Prior to participation in the study, all participants provided their informed consent after being briefed of the study protocol and risks.

Participants included in the study if they were male individuals between the ages of 18 and 27 years, who were students of the University of Kwa-Zulu Natal at the time. Participants who met this initial requirement then completed a physical activity and medical history questionnaire. Participants had to score at least eight points on the FIT (frequency, intensity, time) Index of Kaspari to be considered as moderately active ( $8.58 \pm 1.3 \mathrm{METs}$ ). The FIT Index of Kaspari takes the participants' frequency, intensity, and duration of activity into consideration to provide an estimated level of physical activity. Participants were excluded if they did not meet the above-mentioned criteria, or had diabetes, cardiac disease, smoked, or had hypertension, or were taking any medication that may have influenced vascular tone.

\subsection{Procedures}

Participants were then randomly placed into either the high intensity aerobic interval training group (HIAIT); the steady state aerobic training group (SSAT); or the control group (CG), using randomizer.org. Measurements were conducted at baseline, after three weeks (the mid-point), and after six-weeks (post intervention). 
At baseline, mid-point and post intervention participants were given a 15 minute acclimatization period prior to testing. All testing, assessments, and exercise protocols were carried out in the high performance laboratory based at the Discipline of Biokinetics, Exercise, and Leisure Sciences. Temperature and humidity was regulated and set at 22 degrees Celsius in the laboratory during visits. Lighting was also controlled, and turned off for LDF testing. All participants were asked to refrain from strenuous exercise for 48 hours prior to their laboratory visits, which might have subsequently influenced microvascular reactivity. Participants were asked to fast for at least three hours prior to testing.

During these testing sessions, all participants had their height, weight, body mass index (BMI), resting blood pressure and heart rate, as well as skin fold measurements to determine body fat percentage (BF\%). The participants' baseline skin MVR was then determined. The participants were then allowed a ten minute rest before their $\mathrm{VO}_{2 \max }$, was determined, when finger prick blood lactate samples were also taken.

\subsection{Skin Fold}

Skin fold measurements were taken on the right side of the body using a Harpenden skinfold calliper. Body fat percentage was then calculated using the ACSM seven-site formula: Equation: ACSM seven-site formula: (chest, mid-axillary, triceps, sub-scapular, abdomen, suprailiac, thigh).

Body Density $=1.112-0.00043499$ (Sum of 7 Skinfolds) +0.00000055 (Sum of 7 Skinfolds) $2-0.00028826$ (Age)

\subsection{Laser Doppler Flowmetry}

Laser Doppler Flowmetry is a validated, non-invasive method that utilizes the Doppler shift phenomenon to assess endothelial functioning, an important marker of cardiovascular health (Yvonne-Tee et al., 2005). Skin blood flow (SkBF) was assessed on participants' right palmer forearm, approximately four centimeters distal to the antecubital crease using a moorVMS-LDF, calibrated according to manufacturer's guidelines. The participants were asked to assume a supine position with their right arm immobilized, supinated and resting at heart level, 15 minutes prior to testing, as well as for the duration of the test. Participants were instructed to avoid movement for the duration of the test. A post-occlusive provocation test then took place. A blood pressure cuff was placed on the distal portion of the upper arm, approximately four centimeters distal to the antecubital crease, and was pumped to $50 \mathrm{mmHg}$ above resting systolic blood pressure. Occlusion was maintained for three minutes. The cuff was then be rapidly deflated, and the reactive hyperaemic response was measured. The hyperaemic response time was used as a marker for assessing endothelial function.

Participants of the study were informed that minimal discomfort might occur during the testing. Should a participant have complained of pain, the blood pressure cuff would have been removed immediately, instead of leaving it on for the full three minutes as stated in the protocol. No such complaints were made by any of the participants.

Parameters that were studied were resting flux $(\mathrm{RF})$; maximum increase in hyperaemia perfusion $\left(\mathrm{PORH}_{\max }\right)$; time-to-peak $\left(\mathrm{T}_{\mathrm{p}}\right)$; amplitude of peak perfusion $\left(\mathrm{PORH}_{\text {peak }}\right)$; mean velocity of the hyperaemia response $\left(\mathrm{PORH}_{\max } / \mathrm{T}_{\mathrm{p}}\right)$; and time to resting flux (TRF). Resting flux (RF) is a participant's blood flow at rest. $\mathrm{PORH}_{\max }$ is the maximum increase in post-occlusive reactive hyperaemia perfusion (peak flow above minimum rest flow), expressed as the difference between maximal perfusion flux during post-occlusive reactive hyperaemia and resting flux. $T_{p}$ is the time after occlusion cuff decompression until the post-occlusive peak perfusion flux is reached. $\mathrm{PORH}_{\text {peak }}$ is the amplitude of peak perfusion flux during hyperaemia. $\mathrm{PORH}_{\max } / \mathrm{Tp}$ is the mean velocity of the post-occlusive hyperaemia increase, expressed as a ratio between $\mathrm{PORH}_{\max }$ and $\mathrm{T}_{\mathrm{p}}$. TRF is the time taken for the blood flow (post-occlusion) to reach resting flux. Obtaining TRF provides an optimal model to quantify the participant's' microvascular function from the PORH response; TRF $>7$ seconds is an indicator of endothelial dysfunction (Morales et al., 2005).

\subsection{Maximal Oxygen Consumption $\left(\mathrm{VO}_{2 \max }\right)$}

During the baseline testing session, the participants' maximal aerobic capacity was tested using an electronically braked cycle ergometer (Lode Excalibur Sport, Groningen, The Netherlands). An incremental cycle test was used to obtain gas exchange data using a breath-by-breath gas analyser (Cortex MetaMax $3 \mathrm{~b}$ gas analyser). During the test, the gas samples were averaged every 30 seconds, and the highest values for $\mathrm{VO}_{2}$ and heart rate over 30 seconds were regarded as maximum oxygen uptake $\left(\mathrm{VO}_{2 \max }\right)$ and heart rate (HRmax). Equipment and software was calibrated according to the manufacturer's guidelines. Five second heart rate values were recorded with a HR monitor with the participants wearing a chest belt (S810; Polar, Kempele, Finland).

Time to exhaustion (TE, seconds) was recorded for the test. The test began with a three minute warm-up at 100W, 
followed by an initial $125 \mathrm{~W}$ workload, and $25 \mathrm{~W}$ increments every three minutes in a stepwise fashion. Participants were asked to maintain a minimum pedal cadence of 60 repetitions per minute. At the mid-point (three weeks), and at the end of the six-week period (post intervention) participants' $\mathrm{VO}_{2 \max }$ were reassessed using the same protocol.

\subsection{Blood Lactate Testing}

All lactate samples were taken on the third (middle) or fourth digit of the right and left hands. All samples were analyzed using the Accutrend Plus lactate analyzer (Cobas). Samples were taken at the three testing time points A baseline sample was taken prior to $\mathrm{VO}_{2 \max }$ testing. Samples were then taken at the end of stages of the stepwise protocol, followed by two post-exercise samples taken at minutes three and six following the completion of the $\mathrm{VO}_{2 \max }$ test.

\subsection{Exercise Intervention}

Participants from both the HIAIT and the SSAT groups trained twice a week for six-weeks under supervision in the high performance laboratory on the same cycle ergometer used for the testing procedures. They were allowed a five-minute warm-up prior to exercise. Exercise bouts were determined using the power output produced during the $\mathrm{VO}_{2 \max }$ test. The HIAIT group cycled for 30 second intervals at $100 \%$ of their $\mathrm{VO}_{2 \max }$, followed by three minutes of active rest periods, repeated for six bouts. The SSAT group cycled, for 30 minutes at $60 \%$ of their $\mathrm{VO}_{2 \max }$, with no rest interval. The participants completed a six-week training program. The CG did not exercise for the six-week period. Participants in the HIAIT were required to train twice a week, at $100 \%$ of their $\mathrm{VO}_{2 \max }$. The SSAT participants trained at $60 \%$ of their $\mathrm{VO}_{2 \max }$, twice a week over the six-weeks. The CG only had baseline, midpoint, and post study testing done over the six-week period.

\subsection{Data Analysis}

Data analyses was performed using the IBM SPSS Statistics for Windows software package, Version 23.3.1. IBM Corp., Chicago. Routine descriptive statistics (mean \pm SD) were calculated. The significance of associations between parameters was determined using mixed ANOVA. Laser Doppler parameters were set as the dependent variables and the independent variables included the cardio-respiratory and body composition. Cohen's $d$ effect sizes (ES) were also calculated to determine if the variables were altered by the training intervention and classified by Hopkins (2009) as: $<0.2$ (trivial), $\geq 0.2$ to 0.59 (small), $\geq 0.6$ to 1.19 (medium) and $\geq 1.2$ (large).

\section{Results}

Only 16 (age: $20.93 \pm 5.05$ yrs) of the 25 participants recruited completed the study and were included in the analysis. The attrition was due to unforeseen circumstances that the researchers were unable to control such as participants missing 2 or more consecutive sessions. Table 1 reflects the mean values of the 16 participants' (HIAIT: $\mathrm{n}=7$; SSAT: $\mathrm{n}=3$; CG: $\mathrm{n}=6$ ) height, weight, $\mathrm{VO}_{2 \max }$, and $\mathrm{BF} \%$ at the three testing points in the study. There were no significant changes in these results within the groups.

Table 1. Participant characteristics represented as mean ( \pm standard deviation) at baseline, midpoint, and post-intervention

\begin{tabular}{|c|c|c|c|}
\hline Variable & HIAIT $(n=7)$ & SSAT $(n=3)$ & CG $(n=6)$ \\
\hline Age (years) & $20.43( \pm 1.81)$ & $20.33( \pm 2.309)$ & $22( \pm 2.683)$ \\
\hline Height (m) & $1.73( \pm 0.12)$ & $1.71( \pm 0.005)$ & $1.74( \pm 0.07)$ \\
\hline Weight (kg) (Baseline) & $69.67( \pm 13.27)$ & $75.06( \pm 17.99)$ & $72( \pm 10.62)$ \\
\hline Weight (kg) (Mid-point) & $68.56( \pm 14.15)$ & $74.5( \pm 17.88)$ & $72.28( \pm 11.003)$ \\
\hline Weight (kg) (Post intervention) & $69.37( \pm 13.08)$ & $75.33( \pm 18.55)$ & $72.02( \pm 10.25)$ \\
\hline $\mathrm{VO}_{2 \max }\left(\mathrm{mL} \cdot \mathrm{kg}^{-1} \cdot \mathrm{min}^{-1}\right)$ (Baseline) & $42.12( \pm 8.66)$ & $35( \pm 7.54)$ & $40( \pm 3.08)$ \\
\hline$V_{2 \max }\left(\mathrm{mL} \cdot \mathrm{kg}^{-1} \cdot \mathrm{min}^{-1}\right)($ Mid-point) & $41.1( \pm 8.2)$ & $38.2( \pm 6.00)$ & $39.2( \pm 3.96)$ \\
\hline $\mathrm{VO}_{2 \max }\left(\mathrm{mL} \cdot \mathrm{kg}^{-1} \cdot \mathrm{min}^{-1}\right)($ Post intervention) & $42.71( \pm 8.85)$ & $35.03( \pm 4.78)$ & $40.52( \pm 3.67)$ \\
\hline Body Fat percentage (Baseline) & $7.57( \pm 1.68)$ & $12.73( \pm 12.48)$ & $10.89( \pm 3.17)$ \\
\hline Body Fat percentage (Mid-point) & $7.49( \pm 1.52)$ & $12.48( \pm 11.98)$ & $10.4( \pm 2.48)$ \\
\hline Body Fat percentage (Post intervention) & $6.95( \pm 1.11)$ & $12.57( \pm 11.68)$ & $10.43( \pm 2.48)$ \\
\hline
\end{tabular}

Values reported as mean \pm SD; CG: control group; SSAT: steady state aerobic training; HIAIT: High intensity aerobic interval training; m: meters; kg: kilogram. 
Table 2. Cohen's $d$ effect sizes we calculated from baseline to post intervention

\begin{tabular}{|c|c|c|c|c|c|c|c|c|}
\hline Group & PORH $_{\max }$ & Effect size & $T_{p}$ & Effect size & PORH $_{\max }$ & Effect size & $\mathbf{P O R H}_{\max } / \mathbf{T}_{\mathrm{p}}$ & Effect size \\
\hline HIAIT & 0.155 & Small & 0.632 & Trivial & 0.224 & Small & 0.388 & Small \\
\hline SSAT & 0.080 & Trivial & -0.007 & Trivial & 0.101 & Small & -0.085 & Trivial \\
\hline CG & -0.666 & Trivial & -0.166 & Trivial & -0.670 & Trivial & -0.486 & Trivial \\
\hline
\end{tabular}

CG: control group; SSAT: steady state aerobic training; HIAIT: High intensity aerobic interval training; PORH $_{\text {peak }}$ Post Occlusive Reactive Hyperaemia peak. The peak amplitude post occlusion; $\mathrm{T}_{\mathrm{p}}$ : Time to Peak. The time taken to reach peak amplitude; $\mathrm{PORH}_{\text {peak }} / \mathrm{T}_{\mathrm{p}}$ : The division between post occlusive reactive hyperaemia and the time to peak.

Table 3. Relationship between dependent LDF variables across the groups $(\mathrm{CG}(\mathrm{n}=6)$, SSAT $(\mathrm{n}=3)$, HIAIT $(\mathrm{n}=7))$

\begin{tabular}{|c|c|c|c|c|c|c|c|c|}
\hline \multicolumn{9}{|l|}{$\mathbf{P O R H}_{\max } / \mathbf{T}_{\mathrm{p}}$} \\
\hline CG/DV & $\mathbf{r}$ & p value & SSAT/DV & $\mathbf{r}$ & p value & HIAIT/DV & $\mathbf{r}$ & p value \\
\hline Baseline & 0,364 & 0.501 & Baseline & 0.998 & 0.039 & Baseline & -0.500 & 0.253 \\
\hline Mid-point & 0.126 & 0.811 & Mid-point & 0.992 & 0.083 & Mid-point & -0.295 & 0.520 \\
\hline \multirow[t]{2}{*}{ Post-intervention } & 0.067 & 0.899 & Post intervention & 0.987 & 0.103 & Post intervention & 0.164 & 0.725 \\
\hline & & & PORH $_{\text {max }}$ & & & & & \\
\hline CG/DV & $\mathbf{r}$ & p value & SSAT/DV & $\mathbf{r}$ & p value & HIAIT/DV & $\mathbf{r}$ & p value \\
\hline Baseline & -0.211 & 0.688 & Baseline & 0.999 & 0.032 & Baseline & -0.413 & 0.357 \\
\hline Mid-point & 0.339 & 0.512 & Mid-point & 0.999 & 0.030 & Mid-point & -0.225 & 0.627 \\
\hline \multirow[t]{2}{*}{ Post intervention } & 0.178 & 0.735 & Post intervention & 0.999 & 0.020 & Post intervention & 0.144 & 0.759 \\
\hline & & & PORH $_{\text {peak }}$ & & & & & \\
\hline CG/DV & $\mathbf{r}$ & p value & SSAT/DV & $\mathbf{r}$ & p value & HIAIT/DV & $\mathbf{r}$ & p value \\
\hline Baseline & -0.128 & 0.808 & Baseline & 0.999 & 0.022 & Baseline & -0.415 & 0.355 \\
\hline Mid-point & 0.463 & 0.355 & Mid-point & 1.000 & 0.006 & Mid-point & -0.183 & 0.694 \\
\hline \multirow[t]{2}{*}{ Post intervention } & 0.267 & 0.609 & Post intervention & 1.000 & 0.011 & Post intervention & 0.161 & 0.730 \\
\hline & & & $\mathbf{T}_{\mathbf{p}}$ & & & & & \\
\hline CG/DV & $\mathbf{r}$ & p value & SSAT/DV & $\mathbf{r}$ & p value & HIAIT/DV & $\mathbf{r}$ & p value \\
\hline Baseline & 0.566 & 0.241 & Baseline & -0.708 & 0.499 & Baseline & 0.277 & 0.547 \\
\hline Mid-point & 0.326 & 0.528 & Mid-point & -0.550 & 0.629 & Mid-point & -0.009 & 0.985 \\
\hline Post intervention & 0.315 & 0.542 & Post intervention & -0.619 & 0.575 & Post intervention & 0.357 & 0.431 \\
\hline
\end{tabular}

CG/DV: Control group/dependent variable; SSAT/DV: Steady state aerobic training/dependent variable; HIAIT/DV: High intensity aerobic interval training/dependent variable; $\mathrm{PORH}_{\text {peak }} / \mathrm{T}_{\mathrm{p}}$ : The division between post occlusive reactive hyperaemia and the time to peak; $\mathrm{PORH}_{\max }$ : Post-occlusive reactive hyperaemia perfusion; $\mathrm{PORH}_{\text {peak }}$ : Post-occlusive Reactive Hyperaemia peak. The peak amplitude post occlusion; $\mathrm{T}_{\mathrm{p}}$ : Time to Peak. The time taken to reach peak amplitude.

Table 2 shows the Cohen's $d$ effect sizes and indicated trivial to small changes in all three groups. Table 3 shows the relationship between dependent LDF variables across the groups. Training in both HIAIT and SSAT groups had no effect on $\mathrm{PORH}_{\max }$ across all three of the measurement time points. Analysis also showed that the type of training caused no significant changes in any of the other dependent variables, over the intervention period. The SSAT had a positive linear relationship for $\mathrm{PORH}_{\max } / \mathrm{T}_{\mathrm{p}}$ from baseline to mid-point $(\mathrm{r}=0.992, \mathrm{p}=0.083)$, and post intervention $(\mathrm{r}=0.987, \mathrm{p}=0.103)$. The SSAT also had a linear relationship for $\mathrm{PORH}_{\text {peak }}$ from baseline $(\mathrm{r}=0.999$, $\mathrm{p}=0.22)$ to mid-point $(\mathrm{r}=1, \mathrm{p}=0.006 ;)$ and post intervention $(\mathrm{r}=1, \mathrm{p}=0.011)$. 


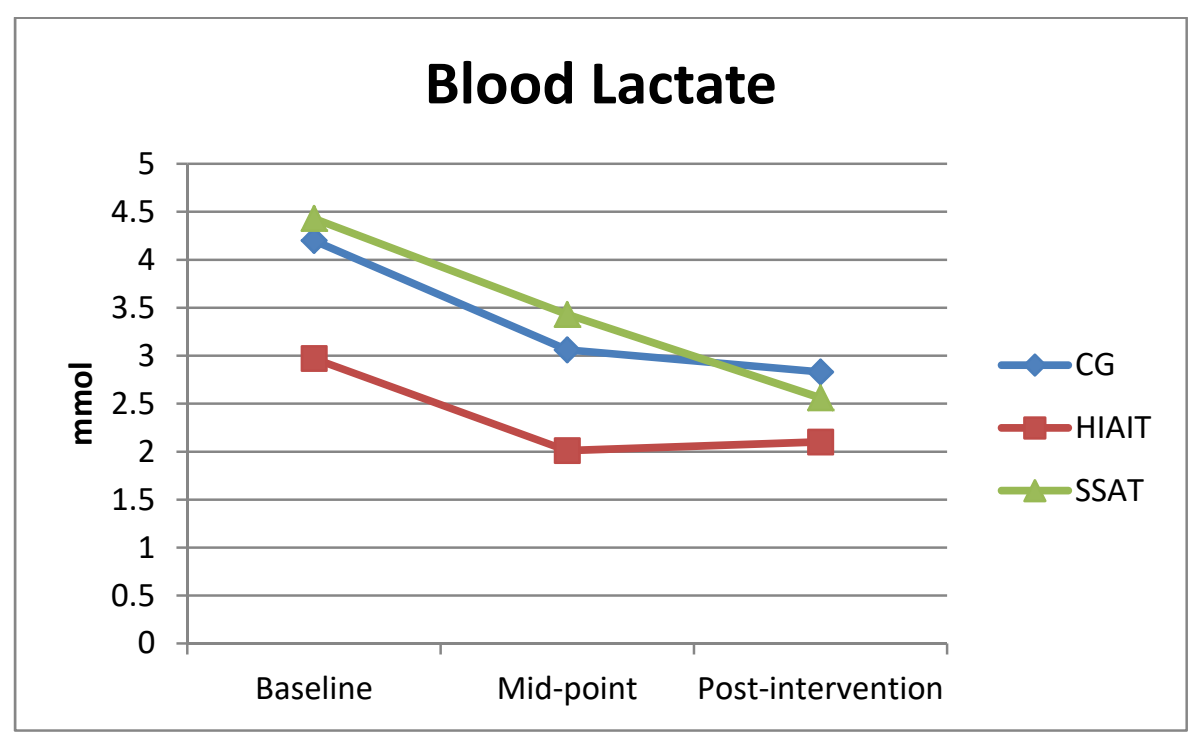

Figure 1. Changes in Blood lactate values over time in the three groups

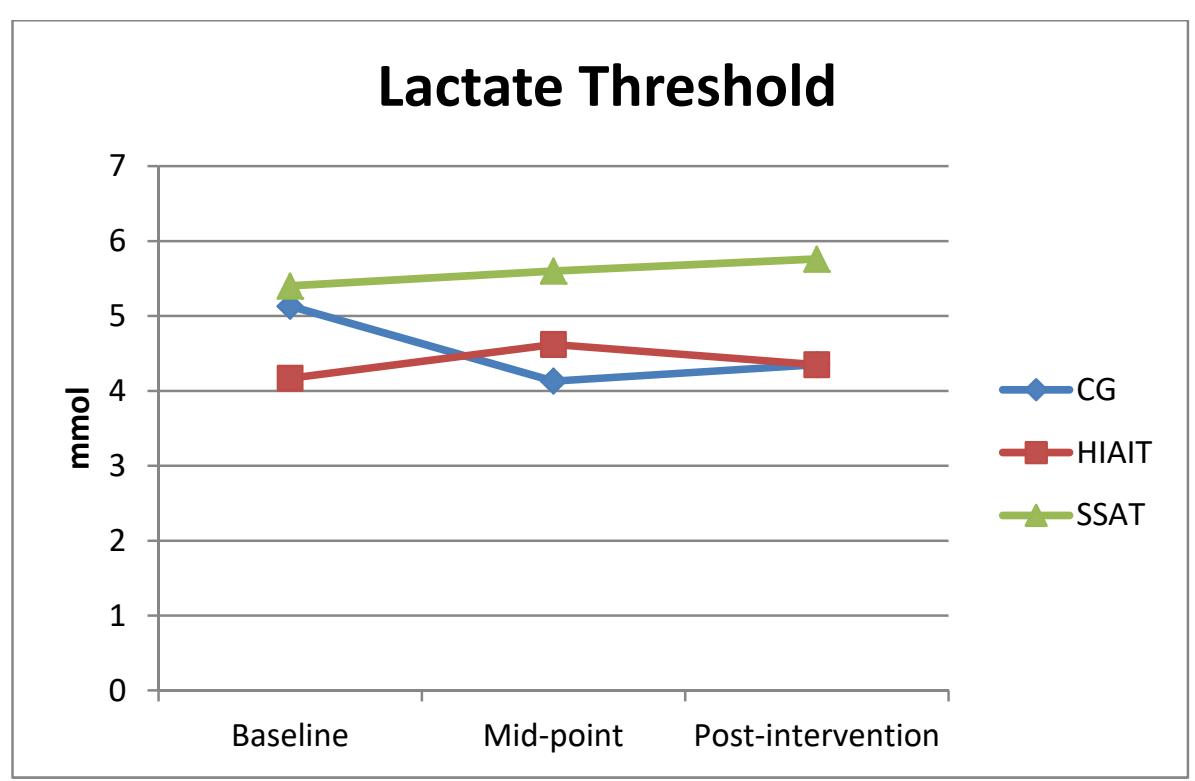

Figure 2. Changes in lactate threshold values over time in the 3 groups 


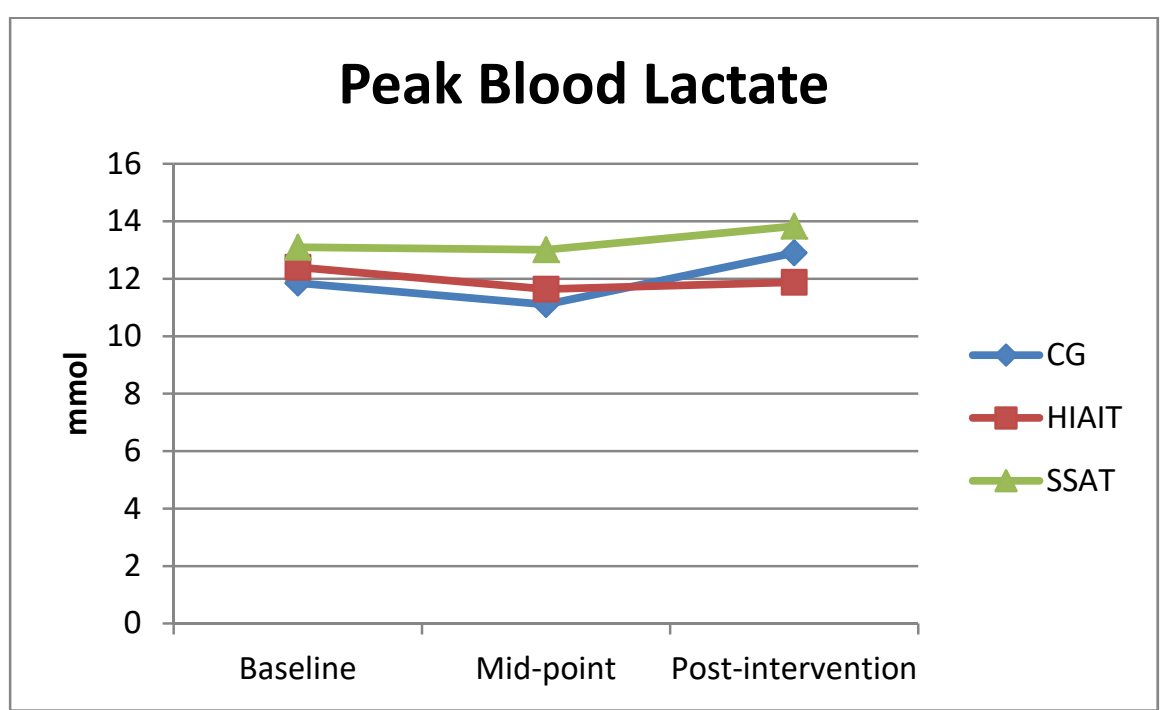

Figure 3. Changes in peak blood lactate values over time in the three groups

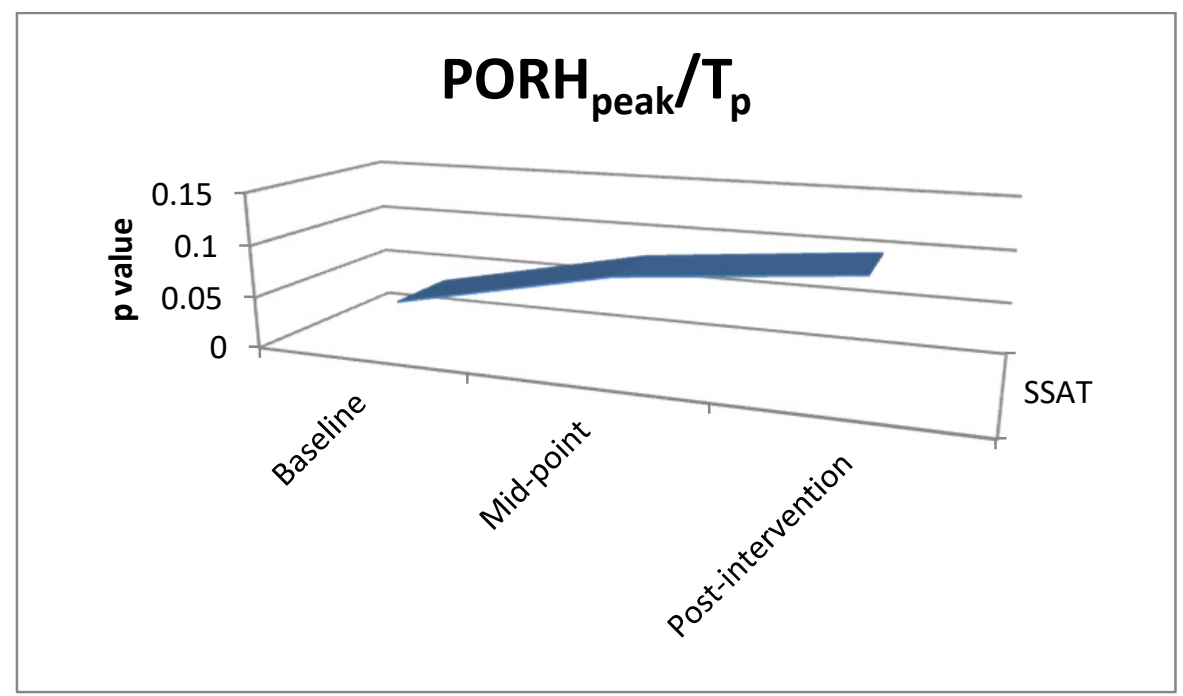

Figure 4. Changes in $\mathrm{PORH}_{\max } / \mathrm{T}_{\mathrm{p}}$ over time in the SSAT group

Figure 1 shows blood lactate changes over time for all three groups. The SSAT group's blood lactate was shown to decrease over time however, these results were not significant $(\mathrm{p}=0.363)$. The SSAT group in this study had also increased both their peak lactate (Figure 2) and their lactate threshold (Figure 3) over time. Figure 4 shows a positive linear relationship between $\mathrm{PORH}_{\max } / \mathrm{T}_{\mathrm{p}}$ in the SSAT group from baseline $(\mathrm{r}=0.998, \mathrm{p}=0.039)$, to mid-point $(r=0.992, p=0.083)$, and then to post intervention $(r=0.987, p=0.103)$.

\section{Discussion}

The main findings of this study indicated that training in either the HIAIT or SSAT groups had no significant effect on skin MVR. However, there was a positive linear relationship with $\mathrm{PORH}_{\text {max }} / \mathrm{T}_{\mathrm{p}}$, as well as $\mathrm{PORH}_{\text {peak }}$ in the SSAT group. This suggests that SSAT may increase the hypereamic response post-occlusion, and may therefore improve overall microvascular response. With regards to blood lactate, no significant change was found across the groups. However, the SSAT group in this study had increased both peak lactate and lactate threshold over the six-week intervention period.

Other studies have found similar results to the present study. A study conducted by Rakobowchuk et al. (2011) concluded that six-weeks of moderate interval training and heavy metabolic interval training did not alter low flow-mediated constriction in healthy participants (Rakobowchuk et al., 2011). Shenouda et al. (2018) tested both 
sedentary men and women using three bouts of all-out cycle sprints for 20 seconds, interspersed with two minutes of active recovery, and found that there was no significant change in brachial artery endothelial function. However, Shenouda et al. (2018) attributed that the low volume used in the study may not have be sufficient to have a significant effect (Shenouda, Skelly, Gibala, MacDonald, 2018). In our study the duration of the intervention (six-weeks) may have not been sufficient to have a significant effect on moderately active individuals.

Previous studies have assessed the effects of cardio-respiratory factors on endothelial functioning and produced results contradicting those in this study. Roche et al. (2008) found that highly trained adolescent soccer players had greater microvascular endothelial vasodilation, as compared to an untrained control group who were matched for age and maturity. In their study, the trained adolescents produced a greater $\mathrm{PORH}_{\text {peak }}$ response as compared to the untrained control group (Roche et al., 2008). A population of young healthy males was tested by Clarkson et al. (1999) in the age group 17 to 24 years. After ten weeks of regular physical activity, which combined both aerobic and anaerobic exercise, the young males showed an improvement in endothelium dependent brachial artery function. Clarkson et al. (1999) attributed the effect of shear stress, a stimulus for nitric oxide (NO) release, as well as the repeated increases in blood flow brought on by regular physical activity as the possible reason for the improvement in endothelial function (Clarkson et al., 1999).

Goto et al. (2003), however, compared the effect of training at different intensities of $\mathrm{VO}_{2 \max }$ on endothelial function in 26 male subjects. They concluded that, after 12 weeks of training, the moderate intensity group (training at $50 \% \mathrm{VO}_{2 \max }$ ), had significantly improved endothelial dependent vasodilation (Goto et al., 2003). Despite our study finding no significant effects on endothelial function, the SSAT did show a positive linear relationships with $\mathrm{PORH}_{\max } / \mathrm{T}_{\mathrm{p}}$, and $\mathrm{PORH}_{\text {peak }}$.

The SSAT group in this study increased both their peak lactate and their lactate threshold. However, conversely in a study comparing HIAIT with high volume training (HVT) in competitive swimmers, Sperlich et al. (2010) showed that swimmers following the HIAIT program improved their performance and their maximal rate of lactate accumulation after five weeks of training (Sperlich et al., 2010).

\section{Limitations}

Due to the time demand of the intervention program and other uncontrollable circumstances the total number of participants as well as participants in each of the groups were low. Due to the randomization process the baseline characteristics between groups was not controlled for. Training frequency was limited to twice weekly and for a period of six-weeks this may not be sufficient to elicit positive effects in moderately active individuals.

\section{Strengths}

The present study provided information on moderately active individuals, most studies have used sedentary individuals or highly trained individuals. The study protocol was well controlled and included three testing time points for comparison. This study's methodology provides a basis for future studies to incorporate exercise interventions to improve endothelial function.

\section{Conclusion}

The results of this study showed that training with HIAIT or SSAT did not have any significant effect on skin MVR. However, the SSAT had a positive linear relationship with $\mathrm{PORH}_{\max } / \mathrm{T}_{\mathrm{p}}$ and $\mathrm{PORH}_{\text {peak. }}$. This could suggest a positive impact of SSAT. Future studies need to incorporate a longer intervention duration with more participants per group.

\section{Competing Interests Statement}

The authors declare that there are no competing or potential conflicts of interest.

\section{References}

Abbott, R. A., Harkness, M. A., \& Davies, P. S. (2002). Correlation of habitual physical activity levels with flow-mediated dilation of the brachial artery in 5-10 year old children. Atherosclerosis, 160, 233-239. https://doi.org/10.1016/S0021-9150(01)00566-4

Celermajer, D. S., Sorensen, K. E., Bull, C., Robinson, J., \& Deanfield, J. E. (1994). Endothelium-dependent dilation in the systemic arteries of asymptomatic subjects relates to coronary risk factors and their interaction. Journal of the American College of Cardiology, 24, 1468-1474. https://doi.org/10.1016/0735-1097(94)90141-4

Clarkson, P., Montgomery, H. E., Mullen, M. J., Donald, A. E., Powe, A. J., Bull, T., ... Deanfield, J. E. (1999) Exercise training enhances endothelial function in young men. Journal of the American College of 
Cardiology, 33, 1379-1385. https://doi.org/10.1016/S0735-1097(99)00036-4

Cohen, N. D., Dunstan, D. W., Robinson, C., Vulikh, E., Zimmet, P. Z., \& Shaw, J. E. (2008). Improved endothelial function following a 14-month resistance exercise training program in adults with type 2 diabetes. Diabetes research and clinical practice, 405-411. https://doi.org/10.1016/j.diabres.2007.09.020

DeSouza, C. A., Shapiro, L. F., Clevenger, C. M., Dinenno, F. A., Monahan, K. D., Tanaka, H., \& Seals, D. R. (2000). Regular aerobic exercise prevents and restores age-related declines in endothelium-dependent vasodilation in healthy men. Circulation, 102, 1351-1357. https://doi.org/10.1161/01.CIR.102.12.1351

Gibala, M. J., Gillen, J. B., \& Percival, M. E. (2014). Physiological and Health-Related Adaptations to Low-Volume Interval Training: Influences of Nutrition and Sex. Sports Med, 44(Suppl 2), S127-S137. https://doi.org/10.1007/s40279-014-0259-6

Goto, C., Higashi, Y., Kimura, M., Noma, K., Hara, K., Nakagawa, K., ... Nara, I. (2003). Effect of Different Intensities of Exercise on Endothelium-Dependent Vasodilation in Humans: Role of Endothelium-Dependent Nitric Oxide and Oxidative Stress. American Heart Association Circulation, 108, 530-535. https://doi.org/10.1161/01.CIR.0000080893.55729.28

Hajer, G. R., van Haeften, T. W., \& Visseren, F. L. J. (2008). Adipose tissue dysfunction in obesity, diabetes, and vascular diseases. European Heart Journal, 29, 2959-2971. https://doi.org/10.1093/eurheartj/ehn387

Hambrecht, R., Adams, V., Erbs, S., \& Linke, A. (2003). Regular physical activity improves endothelial function in patients with coronary artery disease by increasing phosphorylation of endothelial nitric oxide synthase. Circulation, 107, 3152-3158. https://doi.org/10.1161/01.CIR.0000074229.93804.5C

Hopkins, W., Marshall, S., \& Batterham, A. (2009). Progressive statistics for studies in sports medicine and exercise science. Medicine \& Science In Sports \& Exercise, 41, 3-12. https://doi.org/10.1249/MSS.0b013e31818cb278

Horiuchi, M., \& Okita, K. (2012). Blood flow restricted exercise and vascular function. International Journal of Vascular Medicine, 2012, Article ID 543218. https://doi.org/10.1155/2012/543218

Hwang, M. H., \& Lee, S. (2016). Insulin Resistance: Vascular Function and Exercise. Integrative Medicine Research, 5(3). Korea Institute of Oriental Medicine, 198-203. https://doi.org/10.1016/j.imr.2016.06.001

La Favor, J. D., Dubis, G. S., Yan, H., White, J. D., Nelson, M. A. M., Anderson, E. J., \& Hickner, R. C. (2016). 'Microvascular Endothelial Dysfunction in Sedentary, Obese Humans is mediated by NADPH Oxidase; Influence of Exercise Training. Arteriosclerosis Thrombosis Vascular Biology. https://doi.org/10.1161/ATVBAHA.116.308339

Larsen, M. K., \& Matchkov, V. V. (2016). Hypertension and Physical Exercise: The Role of Oxidative Stress. Medicina (Lithuania), 52(1). The Lithuanian University of Health Sciences, 19-27. https://doi.org/10.1016/j.medici.2016.01.005

Montero, D. (2015). The association of cardiorespiratory fitness with endothelial or smooth muscle vasodilator $\begin{array}{llll}\text { function. European Journal of Preventive Cardiology, 22(9), 1200-1211 } & \end{array}$ https://doi.org/10.1177/2047487314553780

Morales, F., Graff, R., Smit, A. J., Bertuglia, S., Petoukhova, A. L., Steenbergen, W., ... Rakhorst, G. (2005). How to assess post-occlusive reactive hyperaemia by means of laser Doppler perfusion monitoring: application of a standardised protocol to patients with peripheral arterial obstructive disease. Microvascular Research, 69, 17-23. https://doi.org/10.1016/j.mvr.2005.01.006

Rajan, V., Varghese, B., van Leeuwen, T., \& Steenbergen, W. (2009). Review of methodological developments in laser Doppler flowmetry. Lasers in medical science, 24, 269-283. https://doi.org/10.1007/s10103-007-0524-0

Rakobowchuk, M., Tanguay, S., Burgomaster, K. A., Howarth, K. R., Gibala, M. J., \& MacDonald, M. J. (2008) 'Sprint interval and traditional endurance training induce similar improvements in peripheral arterial stiffness and flow-mediated dilation in healthy humans. American Journal of Physiology, 295, R236-R242 https://doi.org/10.1152/ajpregu.00069.2008

Roche, D. M., Edmunds, S., Cable, T., Didi, M., \& Stratton, G. (2008). Skin microvascular reactivity in children and adolescents with type 1 diabetes in relation to levels of physical activity and aerobic fitness. Psychology, Health and Medicine, 12(3), 353-363. 
Shenouda, N., Skelly, L. E., Gibala, M. J., \& MacDonald, M. J. (2018). Brachial artery endothelial function is unchanged after acute sprint interval exercise in sedentary men and women. Experimental Physiology. https://doi.org/10.1113/EP086677

Sperlich, B., Zinner, C., Heilemann, I., Kjendlie, P. L., Holmberg, H. C., \& Mester, J. (2010) "High-Intensity Interval Training Improves VO2peak, Maximal Lactate Accumulation, Time Trial and Competition Performance in 9-11-Year-Old Swimmers. European Journal of Applied Physiology, 110(5), 1029-36. https://doi.org/10.1007/s00421-010-1586-4

Tinken, T. M., Thijssen, D. H. J., Black, M. A., Cable, N. T., \& Green, D. J. (2008). Time course of change in vasodilator function and capacity in response to exercise training in humans. Journal of Physiology, 586(20), 5003-5012. https://doi.org/10.1113/jphysiol.2008.158014

Tur, E., Yosipovitch, G., \& Bar-on, Y. (1991). Skin Reactive Hyperemia in Diabetic Patients. DIABETES CARE, 14(11). https://doi.org/10.2337/diacare.14.11.958

Vinet, A., Obert, P., Courteix, D., Chapier, R., Lesourd, B., Verney, J., Dutheil, F., \& Walther, G. (2018). Different Modalities of Exercise Improve Macrovascular Function but Not Microvascular Function in Metabolic Syndrome: The RESOLVE Randomized Trial. International Journal of Cardiology, 267, 165-70. https://doi.org/10.1016/j.ijcard.2018.05.073

Weber, M. A., Hildebrandt, W., Schröder, L., Kinscherf, R., Krix, M., Bachert, P., ... Krakowski-Roosen, H. (2010) Concentric resistance training increases muscle strength without affecting microcirculation. European Journal of Radiology, 614-621. https://doi.org/10.1016/j.ejrad.2008.12.002

Yvonne-Tee, G. B., Rasool, A. H. G., Halim, A. S., \& Rahman, A. R. A. (2005). Reproducibility of different laser Doppler fluximetry parameters of postocclusive reactive hyperemia inhuman forearm skin. Journal of Pharmacological and Toxicological Methods, 52, 286-292. https://doi.org/10.1016/j.vascn.2004.11.003

\section{Copyrights}

Copyright for this article is retained by the author(s), with first publication rights granted to the journal.

This is an open-access article distributed under the terms and conditions of the Creative Commons Attribution license (http://creativecommons.org/licenses/by/4.0/). 